Article

\title{
A Novel and Efficient Five-Component Synthesis of Pyrazole Based Pyrido[2,3-d]pyrimidine-diones in Water: A Triply Green Synthesis
}

\author{
Majid M. Heravi * and Mansoureh Daraie \\ Department of Chemistry, School of Science, Alzahra University, Vanak, Tehran 1993893973, Iran; \\ daraie_m@yahoo.com \\ * Correspondence: mmh1331@yahoo.com; Tel.: +98-21-8804-4040 \\ Academic Editor: Romano V. A. Orru \\ Received: 25 February 2016; Accepted: 24 March 2016; Published: 1 April 2016
}

\begin{abstract}
A novel one pot synthesis of pyrazolo $\left[4^{\prime}, 3^{\prime}: 5,6\right]$ pyrido[2,3-d]pyrimidine-diones, via a five-component reaction, involving, hydrazine hydrate, ethyl acetoacetate, and 1,3-dimethyl barbituric acid, an appropriate aryl aldehydes and ammonium acetate catalyzed via both of heterogeneous and homogeneous catalysis in water, is reported.
\end{abstract}

Keywords: pyrazolo $\left[4^{\prime}, 3^{\prime}: 5,6\right]$ pyrido[2,3-d]pyrimidine-diones; green synthesis; multi-component reaction; in water reaction

\section{Introduction}

Among the known infectious diseases, tuberculosis (TB) [1] and malaria [2] are the most distressing and devastating, both from impermanence and sickness points of view. Malaria and TB are primeval and prolonged infectious diseases initiated chiefly by the parasite Plasmodium falciparum and the pathogen Mycobacterium tuberculosis (MTB), respectively [3,4].

Electron-rich nitrogen heterocycles, such as some pyrimidine derivatives, are well established as antimalarial agents [5]. They also play key roles in diverse biological activities. For instance, a wide range of pyrimidine derivatives show other widespread biological activities and are being used as pharmaceuticals for industrial and prescribed drugs [6]. They show potencies such as Tie-2 kinase inhibitors [7], HIV-1 inhibitors [8], adenosine A1 receptor antagonism [9], anticancer agents [10], analgesics [11], cardiovascular agents and anti-allergic agents [12].

Facile and green synthetic approaches are an important issue in organic synthesis. The generation of divergent and complicated molecules from either commercially available or readily accessible starting materials is an inspiring theme in contemporary organic synthesis [13]. The compatible combination of multi-component reactions (MCRs) and unconventional reaction conditions has recently attracted much attention and stirred up the interest of synthetic organic chemists, resulting in concurrent advance and growth of both MCRs and green chemistry toward ideal synthetic chemistry [14,15]. Multi-component reactions (MCRs), in which multiple reactions are joined in a single synthetic operation performed in one pot, have been extensively and broadly employed in the total synthesis of natural products and their building blocks [16]. Using such strategies for the synthesis of a desired target avoids purification of different precursors as well as tedious protection and deprotection of functional groups frequently, required in multistep synthesis. Due to the shortening of the reaction steps and atom economy involved, a higher degree of component-multiplicity leads to greener conditions.

Nowadays, green chemistry has become a main motivation and inspiration for organic chemists to develop eco-friendly and benign pathways for the synthesis of organic compounds, particularly 
those exhibiting biological activity [17,18]. Manipulation of multi-component reactions, performed in water as a green and abundant solvent, is another attractive concern of organic synthetic chemists, complying with green chemistry principles. A wide variety of divergent chemical transformations can occur in water. Breslow [19], Li [20], Kobayashi [21], Sharpless [22] and many other renowned organic chemists have momentously extended the number of reactions that can be conducted in water. Reaction in water offers unique reactivity and selectivity. Moreover, water can be readily separated and isolated from organic materials by simple procedures.

Another latent opportunity for in water synthesis is the development of a high atom economy that can facilitate catalyst recovery, recycling and simple product isolation. Idyllically, the reactant and the product should have no or very little water-solubility. Thus, the product can be isolated by simple phase separations and the catalyst can be easily recycled. As a matter of fact, in such circumstances, it is possible to recycle the catalyst-bearing aqueous solution for a prolonged period of time without the requirement either discharging it or recreating it, providing the low atom-efficiency process that resulted in the accretion of undesired materials in the solution.

Heterogeneous catalysis is of paramount importance in green chemistry, due to simplicity of separation, recovery and recyclability of most heterogeneous catalysts [23,24].

Pyrazolo based pyrido[2,3- $d$ ] pyrimidine-diones and their derivatives are a very important class of heterocycles with a wide range of pharmacological and biological activities.

The synthesis of pyrazole based pyrido[2,3-d]pyrimidine-diones was reported in 2012 and 2014 via a three component reaction using barbituric acids, $1 H$-pyrazol-5-amines and differently substituted aromatic aldehydes using PTSA ( $p$-Toluenesulfonic acid) under solvent-free conditions, IL-cells (cellulose supported ionic liquid) and ethanol have been reported respectively $[25,26]$.

We are interested in heterocyclic chemistry and chemical and molecular diversity in this field [27-34]. In continuation of our investigation on the synthesis of heterocyclic compounds using different heterogeneous nanocatalysts in organic reactions and, in particular, different heterocyclic systems [35-44], and expansion of our work on multi-component reactions and green chemistry [45-54], herein, we report an efficient triply green protocol for the synthesis of pyrazole based pyrido[2,3- $d]$ pyrimidine-diones.

\section{Results}

Initially, we designed a one pot four-component reaction, employing different starting materials leading to pyrazole based pyrido[2,3-d]pyrimidine-diones in the absence of any catalyst in water (Scheme 1).

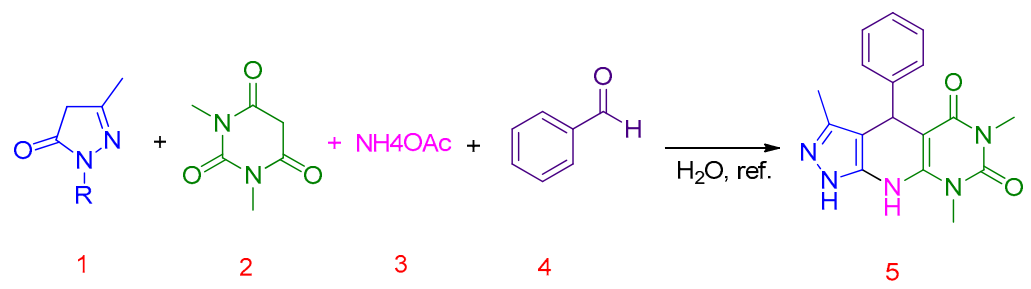

Scheme 1. Model reaction for pyrazole based pyrido[2,3-d]pyrimidine-dione synthesis.

In this strategy, as a model reaction, we used 3-methyl-pyrazolone 1, 1,3-dimethyl barbituric acid 2, ammonium acetate 3 and benzaldehyde 4 in refluxing water. This reaction was monitored by TLC (Thin layer chromatography), showing no progress.

Thus, we examined the reaction in the presence of various catalysts in different solvents under mild reaction conditions (Table 1). 
Table 1. Optimization of the reaction conditions for the synthesis of pyrazole based pyrido[2,3- $d]$ pyrimidine-diones under thermal conditions.

\begin{tabular}{ccccc}
\hline Entry & Catalyst & Solvent & Time (h) & Yield (\%) \\
\hline 1 & $\mathrm{H}_{6} \mathrm{P}_{2} \mathrm{~W}_{18} \mathrm{O}_{62} \cdot 18 \mathrm{H}_{2} \mathrm{O}$ & Water & 2 & 60 \\
2 & Sulfamic acid & Water & 2.5 & 65 \\
3 & DABCO & Water & 3.5 & 85 \\
4 & $\mathrm{ZnO}$ & Water & 5 & 80 \\
4 & Nano- $\mathrm{ZnO}$ & Water & 0.66 & 94 \\
5 & Nano-ZnO & EtOH & 1 & 80 \\
\hline
\end{tabular}

Considering this point that has now been turned into a fact, nowadays, a plethora of nanometal oxides are used as efficient catalysts in several of the organic transformations. Among other catalysts examined, we initially used $\mathrm{ZnO}$ and then selected nano- $\mathrm{ZnO}$ from analyzing our results obtained from sets of reactions, as a green catalyst. We have also found that the amount of our model reaction, and, in general, $0.04 \mathrm{~g}$ of nano- $\mathrm{ZnO}$ is the optimum amount of catalyst. Under optimized reaction conditions, using optimal amounts of nano- $\mathrm{ZnO}$ as heterogeneous catalyst in boiling water as solvent, we designed this four component reaction, providing the corresponding pyrazolo-[ $\left.4^{\prime}, 3^{\prime}: 5,6\right]$ pyrido[2,3- $\left.d\right]$ pyrimidine-diones in high yield.

As far as green chemistry is concerned, our methodology was superior than that of the preparation of pyrazole based pyrido[2,3- $d$ ]pyrimidine-diones, using PTSA, previously [25].

When PTSA is heated with acid and water, a hydrolysis reaction takes place and toluene is formed along sulphuric acid.

Our strategy also looks more promising when compared with the recently reported synthesis of pyrazole based pyrido[2,3-d]pyrimidine-diones, using pre-prepared IL-Cells [26] as catalyst, due to the commercial availability of nano- $\mathrm{ZnO}$.

The reaction pathway for both strategies are the same and depicted in Figure 1.

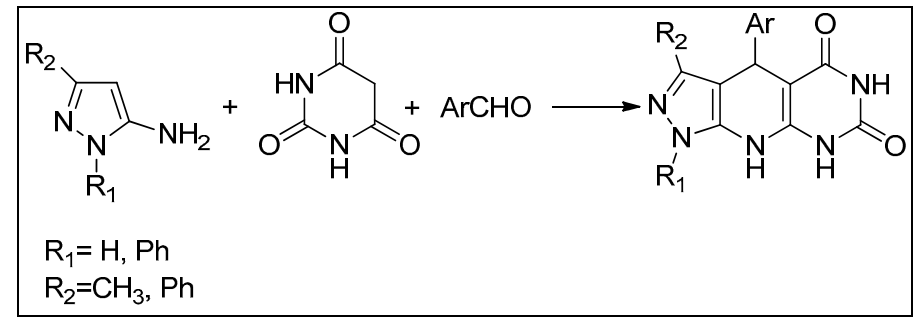

Figure 1. Synthesis of pyrazole based pyrido[2,3- $d$ ]pyrimidine-diones $[25,26]$.

Under optimized conditions, using nano- $\mathrm{ZnO}$ as heterogeneous catalyst in boiling water as solvent, this four component reaction gave the corresponding pyrazolo- $\left[4^{\prime}, 3^{\prime}: 5,6\right]$ pyrido[2,3- $\left.d\right]$ pyrimidine-diones in excellent yield (Table 2).

Table 2. One pot, four-component synthesis of pyrazolo- $\left[4^{\prime}, 3^{\prime}: 5,6\right]$ pyrido[2,3- $\left.d\right]$ pyrimidine-diones in refluxing water using a catalytic amount of nano- $\mathrm{ZnO}$.

\begin{tabular}{|c|c|c|c|c|c|c|}
\hline Entry & Ar & $\mathbf{R}$ & Time (h) & Yield (\%) ${ }^{a}$ & M.p. $\left({ }^{\circ} \mathrm{C}\right)$ & M.p. rep. $\left({ }^{\circ} \mathrm{C}\right)[26]$ \\
\hline 1 & $\mathrm{C}_{6} \mathrm{H}_{5}$ & $\mathrm{H}$ & 3 & 94 & 194-196 & 195-196 \\
\hline 2 & $4-\mathrm{FC}_{6} \mathrm{H}_{4}$ & $\mathrm{H}$ & 2.5 & 90 & $281-283$ & - \\
\hline 3 & $4-\mathrm{ClC}_{6} \mathrm{H}_{4}$ & $\mathrm{H}$ & 2.5 & 94 & $285-286$ & $284-285$ \\
\hline 4 & $4-\mathrm{BrC}_{6} \mathrm{H}_{4}$ & $\mathrm{H}$ & 2.8 & 92 & $286-288$ & $286-287$ \\
\hline 5 & $2,4-\mathrm{ClC}_{6} \mathrm{H}_{3}$ & $\mathrm{H}$ & 3.2 & 92 & $285-287$ & $286-287$ \\
\hline 6 & $4-\mathrm{NO}_{2} \mathrm{C}_{6} \mathrm{H}_{4}$ & $\mathrm{H}$ & 3 & 94 & $172-173$ & 173-174 \\
\hline 7 & $3-\mathrm{NO}_{2} \mathrm{C}_{6} \mathrm{H}_{4}$ & $\mathrm{H}$ & 3.2 & 91 & $221-223$ & $220-221$ \\
\hline
\end{tabular}


Table 2. Cont

\begin{tabular}{ccccccc}
\hline Entry & $\mathbf{A r}$ & $\mathbf{R}$ & Time (h) & Yield (\%) & a.p. $\left({ }^{\circ} \mathbf{C}\right)$ & M.p. rep. $\left({ }^{\circ} \mathbf{C}\right)[\mathbf{2 6}]$ \\
\hline $\mathbf{8}$ & $4-\mathrm{OHC}_{6} \mathrm{H}_{4}$ & $\mathrm{H}$ & 4 & 87 & $220-222$ & $219-220$ \\
$\mathbf{9}$ & $4-\mathrm{N}\left(\mathrm{Me}_{2} \mathrm{C}_{6} \mathrm{H}_{4}\right.$ & $\mathrm{H}$ & 3.8 & 89 & $222-224$ & $221-223$ \\
$\mathbf{1 0}$ & $\mathrm{C}_{6} \mathrm{H}_{5}$ & $\mathrm{Ph}$ & 3.5 & 92 & $208-210$ & $210-211$ \\
$\mathbf{1 1}$ & $4-\mathrm{FC}_{6} \mathrm{H}_{4}$ & $\mathrm{Ph}$ & 3 & 89 & $207-208$ & $208-210$ \\
$\mathbf{1 2}$ & $4-\mathrm{ClC}_{6} \mathrm{H}_{4}$ & $\mathrm{Ph}$ & 3 & 94 & $200-202$ & - \\
$\mathbf{1 3}$ & $4-\mathrm{BrC}_{6} \mathrm{H}_{4}$ & $\mathrm{Ph}$ & 3 & 92 & $160-161$ & $158-159$ \\
$\mathbf{1 4}$ & $4-\mathrm{NO}_{2} \mathrm{C}_{6} \mathrm{H}_{4}$ & $\mathrm{Ph}$ & 3.7 & 88 & $155-157$ & $156-157$ \\
$\mathbf{1 5}$ & $4-\mathrm{CH}_{3} \mathrm{C}_{6} \mathrm{H}_{4}$ & $\mathrm{Ph}$ & 4 & 90 & $205-506$ & $207-209$ \\
\hline
\end{tabular}

Although the literature survey discloses a plethora of multi-component reactions, five-component reactions are scarcely found [55-61].

During our practical work, we observed that the reaction of ethyl acetoacetate and hydrazine hydrate proceeded smoothly and was completed rapidly [62,63] to afford 3-methyl-5-hydrazolone, virtually in quantitative yield. Thus, we decided to investigate the synthesis of pyrazolo- $\left[4^{\prime}, 3^{\prime}: 5,6\right]$ pyrido[2,3-d]pyrimidine-diones via a one pot five component reaction (Scheme 2).

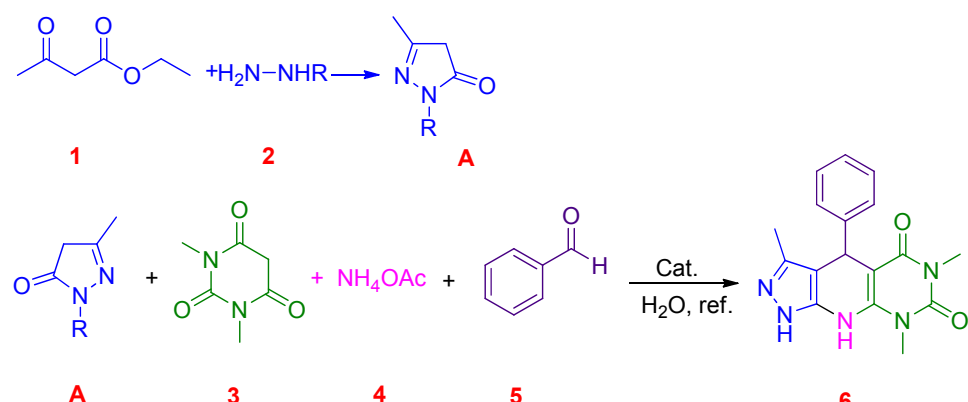

Scheme 2. Five-component reaction for pyrazole based pyrido[2,3-d]pyrimidine-dione synthesis.

The proposed mechanism for a five-component reaction is summarized in Scheme 3.

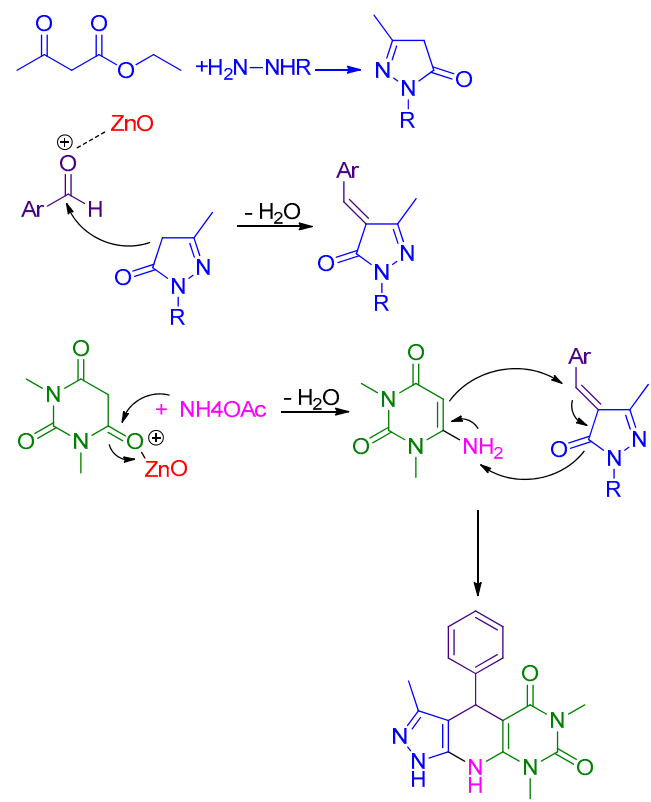

Scheme 3. Proposed mechanism for nano $\mathrm{ZnO}$ catalyst. 
For experimental purposes, initially, hydrazine hydrate $(1.1 \mathrm{mmol})$ was added drop wise to ethyl acetoacetate ( $1 \mathrm{mmol}$ ) and then an already prepared mixture of nano $\mathrm{ZnO}$, benzaldehyde, 1,3-dimethyl barbituric acid and ammonium acetate was added. This mixture was refluxed in water. The progress of our model reaction was monitored by TLC. Upon completion of the reaction, the corresponding pyrazolo- $\left[4^{\prime}, 3^{\prime}: 5,6\right]$ pyrido[2,3-d]pyrimidine-dione was obtained in high yield. (Table 3).

Table 3. One pot, five-component synthesis of pyrazolo- $\left[4^{\prime}, 3^{\prime}: 5,6\right]$ pyrido[2,3- $\left.d\right]$ pyrimidine-diones in refluxing water using a catalytic amount of nano- $\mathrm{ZnO}$.

\begin{tabular}{|c|c|c|c|c|c|c|}
\hline Entry & Ar & $\mathbf{R}$ & Time (h) & Yield (\%) ${ }^{a}$ & M.p. $\left({ }^{\circ} \mathrm{C}\right)$ & M.p. rep. $\left({ }^{\circ} \mathrm{C}\right)[26]$ \\
\hline 1 & $\mathrm{C}_{6} \mathrm{H}_{5}$ & $\mathrm{H}$ & 4 & 91 & 194-196 & 195-196 \\
\hline 2 & $4-\mathrm{FC}_{6} \mathrm{H}_{4}$ & $\mathrm{H}$ & 3.5 & 90 & $281-283$ & - \\
\hline 3 & $4-\mathrm{ClC}_{6} \mathrm{H}_{4}$ & $\mathrm{H}$ & 3.5 & 91 & $285-286$ & $284-285$ \\
\hline 4 & $4-\mathrm{BrC}_{6} \mathrm{H}_{4}$ & $\mathrm{H}$ & 3.8 & 90 & $286-288$ & $286-287$ \\
\hline 5 & $2,4-\mathrm{ClC}_{6} \mathrm{H}_{3}$ & $\mathrm{H}$ & 4 & 90 & $285-287$ & $286-287$ \\
\hline 6 & $4-\mathrm{NO}_{2} \mathrm{C}_{6} \mathrm{H}_{4}$ & $\mathrm{H}$ & 4 & 91 & $172-173$ & $173-174$ \\
\hline 7 & $3-\mathrm{NO}_{2} \mathrm{C}_{6} \mathrm{H}_{4}$ & $\mathrm{H}$ & 4.2 & 89 & $221-223$ & $220-221$ \\
\hline 8 & $4-\mathrm{OH}-\mathrm{C}_{6} \mathrm{H}_{4}$ & $\mathrm{H}$ & 4.5 & 85 & $220-222$ & $219-220$ \\
\hline 9 & $4-\mathrm{N}(\mathrm{Me})_{2} \mathrm{C}_{6} \mathrm{H}_{4}$ & $\mathrm{H}$ & 4.5 & 85 & $222-224$ & $221-223$ \\
\hline
\end{tabular}

The product was deposited in the water to form a separate filtrate, thus consuming much energy to evaporate the solvent being non-required. Upon filtering of the cold reaction mixture, the Zno nano-catalyst remains on the top of funnel along with product. Upon recrystallization of the product from $\mathrm{CH}_{2} \mathrm{Cl}_{2}$, it can be recovered, recycled and reused with a simple washing up, at least in three consecutive runs without appreciable loss of activity.

As is clear from the structure, there is a chiral center in the final product. Since our suggested mechanism involves sequential Knoevenagel-Michael addition reactions that can be catalyzed in both acidic and basic conditions, we thought it was worthwhile to examine asymmetric synthesis of pyrazolo- $\left[4^{\prime}, 3^{\prime}: 5,6\right]$ pyrido[2,3- $\left.d\right]$ pyrimidine-diones via asymmetric organocatalysis.

L-proline is a readily obtainable naturally occurring amino acid. It has also been reported as an eco-friendly catalyst for the synthesis of several heterocycles [64-66]. We thought it was worthwhile to examine it as an asymmetric basic organocatalyst to attain optical activity in our products.

Since several asymmetric synthesis using L-proline has been done at room temperature [67], initially, the model reaction was performed at $25^{\circ} \mathrm{C}$ to afford compound $6 \mathbf{a}$. Unfortunately, the yield of the product was very low, so the reaction mixture was heated at reflux temperature for the indicated times. Although the reaction proceeded smoothly leading to the desired product, we did not observe any optical activity in the isolated product, perhaps due to reflux conditions. To establish the generality of proline-catalyzed reaction, various aromatic aldehydes were used to give the desired products it worked as an ordinary base (Scheme 4). However, the results obtained from the nano-ZnO-catalyzed reaction had overshadowed this approach.

The corresponding product was produced in high yield, but unfortunately does not show any optical activity. The results are summarized in Table 4. 
<smiles>[R]Cn1c(C)nn([R])c1=O</smiles><smiles>CN1C(=O)CC(=O)N(C)C1=O</smiles>

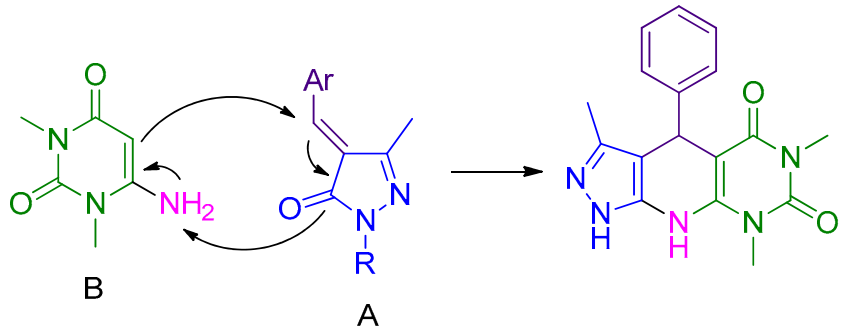

Scheme 4. Proposed mechanism for the synthesis of compound 6 using L-proline.

Table 4. One pot, four-component synthesis of pyrazolo- $\left[4^{\prime}, 3^{\prime}: 5,6\right]$ pyrido[2,3- $\left.d\right]$ pyrimidine-diones in refluxing water using a catalytic amount of nano- $\mathrm{ZnO}$.

\begin{tabular}{|c|c|c|c|c|c|c|}
\hline Comp. & Ar & $\mathbf{R}$ & Time (h) & Yield $(\%)^{a}$ & M.p. $\left({ }^{\circ} \mathrm{C}\right)$ & M.p. rep. $\left({ }^{\circ} \mathrm{C}\right)[26]$ \\
\hline 1 & $\mathrm{C}_{6} \mathrm{H}_{5}$ & $\mathrm{H}$ & 4 & 91 & 195-197 & 195-196 \\
\hline 2 & $4-\mathrm{FC}_{6} \mathrm{H}_{4}$ & $\mathrm{H}$ & 3.5 & 90 & $280-281$ & - \\
\hline 3 & $4-\mathrm{ClC}_{6} \mathrm{H}_{4}$ & $\mathrm{H}$ & 3.5 & 91 & $283-285$ & $284-285$ \\
\hline 4 & $4-\mathrm{BrC}_{6} \mathrm{H}_{4}$ & $\mathrm{H}$ & 4 & 90 & $284-286$ & $286-287$ \\
\hline 5 & $2,4-\mathrm{ClC}_{6} \mathrm{H}_{3}$ & $\mathrm{H}$ & 4.5 & 89 & $286-288$ & $286-287$ \\
\hline 6 & $4-\mathrm{NO}_{2} \mathrm{C}_{6} \mathrm{H}_{4}$ & $\mathrm{H}$ & 4.2 & 90 & $171-173$ & $173-174$ \\
\hline 7 & $3-\mathrm{NO}_{2} \mathrm{C}_{6} \mathrm{H}_{4}$ & $\mathrm{H}$ & 4.5 & 88 & $220-223$ & $220-221$ \\
\hline 8 & $4-\mathrm{OHC}_{6} \mathrm{H}_{4}$ & $\mathrm{H}$ & 5 & 84 & $218-221$ & $219-220$ \\
\hline 9 & $4-\mathrm{N}(\mathrm{Me})_{2} \mathrm{C}_{6} \mathrm{H}_{4}$ & $\mathrm{H}$ & 4.2 & 86 & $223-224$ & $221-223$ \\
\hline 10 & $\mathrm{C}_{6} \mathrm{H}_{5}$ & $\mathrm{Ph}$ & 6 & 89 & $209-212$ & $210-211$ \\
\hline 11 & $4-\mathrm{FC}_{6} \mathrm{H}_{4}$ & $\mathrm{Ph}$ & 5.5 & 87 & $207-209$ & $208-210$ \\
\hline 12 & $4-\mathrm{ClC}_{6} \mathrm{H}_{4}$ & $\mathrm{Ph}$ & 5.5 & 90 & 201-204 & - \\
\hline 13 & $4-\mathrm{BrC}_{6} \mathrm{H}_{4}$ & $\mathrm{Ph}$ & 6 & 90 & 157-159 & $158-159$ \\
\hline 14 & $4-\mathrm{NO}_{2} \mathrm{C}_{6} \mathrm{H}_{4}$ & $\mathrm{Ph}$ & 5.2 & 84 & $154-157$ & $156-157$ \\
\hline 15 & $4-\mathrm{CH}_{3} \mathrm{C}_{6} \mathrm{H}_{4}$ & $\mathrm{Ph}$ & 5.5 & 85 & $206-508$ & 207-209 \\
\hline
\end{tabular}

\section{Experimental}

Melting points were measured by using the capillary tube method with an electro thermal 9200 apparatus. ${ }^{1} \mathrm{H}-\mathrm{NMR}$ and ${ }^{13} \mathrm{C}-\mathrm{NMR}$ spectra were recorded on a Bruker spectrometer (Ettlingen, Germany) at $400 \mathrm{MHz}$, respectively, using TMS (Tetramethylsilane) as an internal standard (DMSO solution). IR spectra were recorded from KBr disk on the FT-IR spectrometer (Ettlingen, Germany) Bruker Tensor 27. The reactions were monitored by TLC. All solvents and reagents were purchased 
from Aldrich (Taufkirchen, Germany) and Merck (Darmstat, Germany) with high-grade quality, and used without any purification. All products are new and were fully characterized by their spectral and physical data (Please find Supplementary Materials for more details).

\subsection{General Procedure}

3.1.1. Synthesis of Pyrazolo-[ $\left[4^{\prime}, 3^{\prime}: 5,6\right]$ pyrido[2,3- $\left.d\right]$ pyrimidine-diones: Using 4-Component Reaction

To a mixture of 3-methyl-pyrazol-5-one or 3-methyl-1-phenyl-pyrazole-5-one (1 mmol), 1,3-dimethyl barbitueic acid $(1 \mathrm{mmol})$, ammonium acetat $(1.2 \mathrm{mmol})$ and benzaldehyde $(1 \mathrm{mmol})$, a catalytic amount of nano- $\mathrm{ZnO}(0.04 \mathrm{~g})$ was added and the resulting mixture was heated at reflux in $\mathrm{H}_{2} \mathrm{O}(5 \mathrm{~mL})$. The progress of the reaction was monitored by TLC. On completion, the mixture was cooled and filtered. The precipitate was recrystallized from $\mathrm{CH}_{2} \mathrm{Cl}_{2}$ to give pure target compounds. All the products were identified by comparison of their physical and spectroscopic data with those reported for authentic samples. The physical and spectral data are given.

\subsubsection{Synthesis of Pyrazolo-[ $\left[4^{\prime}, 3^{\prime}: 5,6\right]$ pyrido[2,3-d]pyrimidine-diones Using 5-Component Reaction}

Initially, hydrazine hydrate $(1.1 \mathrm{mmol})$ was added drop wise to ethyl acetoacetate $(1 \mathrm{mmol})$ and then other components containing 1,3-dimethyl barbitueic acid ( $1 \mathrm{mmol})$, ammonium acetat (1.2 $\mathrm{mmol})$, benzaldehyde ( $1 \mathrm{mmol}), \mathrm{ZnO}$ nanoparticles $(0.04 \mathrm{~g})$ were added at once, and the mixture was heated at reflux temperature for the time indicated in Table 3. After completion of the reaction (as monitored by TLC), the mixture was cooled and filtered. The precipitate was recrystallized from $\mathrm{CH}_{2} \mathrm{Cl}_{2}$ to give pure target compounds.

\subsubsection{Synthesis of Pyrazolo-[ $\left[4^{\prime}, 3^{\prime}: 5,6\right]$ pyrido[2,3-d]pyrimidine-diones: Using 4-Component Reaction}

A solution of 3-methyl-pyrazol-5-one or 3-methyl-1-phenyl-pyrazole-5-one (1 mmol), 1,3-dimethyl barbitueic acid $(1 \mathrm{mmol})$, ammonium acetat $(1.2 \mathrm{mmol})$, benzaldehyde $(1 \mathrm{mmol})$ and L-proline $(0.04 \mathrm{~g})$ in $\mathrm{H}_{2} \mathrm{O}(5 \mathrm{~mL})$ was stirred under heating conditions for appropriate time. After completion of the reaction which was monitored by TLC, the mixture was cooled to room temperature. The solid product was collected by filtration, washed with water and aqueous ethanol and purified by recrystallization from $\mathrm{CH}_{2} \mathrm{Cl}_{2}$.

\subsection{Spectral Data}

3,6,8-Trimethyl-4-phenyl-8,9-dihydro-1H-pyrazolo[4' ,3':5,6]pyrido[2,3-d]pyrimidine-5,7(4H,6H)-dione (Entry 1, Table 2). m.p.: $194-196^{\circ} \mathrm{C}$; IR (KBr, $\left.v_{\max }, \mathrm{cm}^{-1}\right)$ : 3332, 2971, 1654, $1248 .{ }^{1} \mathrm{H}-\mathrm{NMR}\left(400 \mathrm{MHz}, \mathrm{DMSO}-d_{6}\right)$ : $\delta 2.22\left(\mathrm{~s}, 3 \mathrm{H}, \mathrm{CH}_{3}\right), 2.81\left(\mathrm{~s}, 3 \mathrm{H}, \mathrm{CH}_{3}\right), 3.07\left(\mathrm{~s}, 3 \mathrm{H}, \mathrm{CH}_{3}\right), 5.63(\mathrm{~s}, 1 \mathrm{H}, \mathrm{CH}), 7.006-7.084(\mathrm{~m}, 3 \mathrm{H}, \mathrm{Ar}-\mathrm{H})$, 7.158-7.196 (t, 2H, J = 7.2, Ar-H), 10.46 (brs, 2H, 2-NH).

4-(4-Fluorophenyl)-3,6,8-trimethyl-8,9-dihydro-1H-pyrazolo[4', 3':5,6]pyrido[2,3-d]pyrimidine-5,7(4H,6H)-dione (Entry 2, Table 2). m.p.: $281-283^{\circ} \mathrm{C}$; IR (KBr, $\left.v_{\max }, \mathrm{cm}^{-1}\right)$ : 3312, 2956, 1663, 1280. ${ }^{1} \mathrm{H}-\mathrm{NMR}(400 \mathrm{MHz}$, DMSO- $\left.d_{6}\right): \delta 1.96\left(\mathrm{~s}, 3 \mathrm{H}, \mathrm{CH}_{3}\right), 3.06\left(\mathrm{~s}, 3 \mathrm{H}, \mathrm{CH}_{3}\right), 3.46\left(\mathrm{~s}, 3 \mathrm{H}, \mathrm{CH}_{3}\right), 4.99(\mathrm{~s}, 1 \mathrm{H}, \mathrm{CH}), 7.14-7.16(\mathrm{~d}$, $J=7.6 \mathrm{~Hz}, 2 \mathrm{H}, \mathrm{Ar}-\mathrm{H}), 7.36-7.38(\mathrm{~d}, J=7.6 \mathrm{~Hz}, 2 \mathrm{H}, \mathrm{Ar}-\mathrm{H}), 9.81(\mathrm{~s}, 1 \mathrm{H}, \mathrm{NH}), 11.99(\mathrm{~s}, 1 \mathrm{H}, \mathrm{NH}) ;{ }^{13} \mathrm{C}-\mathrm{NMR}$ $\left(100 \mathrm{MHz}\right.$, DMSO- $\left.d_{6}\right) \delta: 10.02,27.15,30.28,33.84,88.63,106.23,125.06,130.41,134.57,135.63,137.31$, $142.28,149.62,150.19,158.24$; calcd for $\mathrm{C}_{17} \mathrm{H}_{16} \mathrm{FN}_{5} \mathrm{O}_{2}$ : C, 57.07; H, 4.51; N, 19.57; found: C, 57.12; $\mathrm{H}, 4.50 ; \mathrm{N}, 20.02$

4-(4-Chlorophenyl)-3,6,8-trimethyl-8,9-dihydro-1H-pyrazolo[4', 3':5,6]pyrido[2,3-d]pyrimidine-5,7(4H,6H)-dione (Entry 3, Table 2). m.p.: $285-285^{\circ} \mathrm{C}$; IR (KBr, $\left.v_{\max }, \mathrm{cm}^{-1}\right): 3321,2948,1647,1263$.

4-(4-Bromophenyl)-3,6,8-trimethyl-8,9-dihydro-1H-pyrazolo[4', 3':5,6]pyrido[2,3-d]pyrimidine-5,7(4H,6H)-dione (Entry 4, Table 2). m.p.: $286-288^{\circ} \mathrm{C}$; IR (KBr, $\left.v_{\max }, \mathrm{cm}^{-1}\right): 3329,2948,1644,1261$. 
4-(2,4-Dichlorophenyl)-3,6,8-trimethyl-8,9-dihydro-1H-pyrazolo[4', 3':5,6]pyrido[2,3-d]pyrimidine-5,7(4H,6H)dione (Entry 5, Table 2). m.p.: 285-287 ${ }^{\circ} \mathrm{C}$; IR (KBr, $\left.v_{\max }, \mathrm{cm}^{-1}\right)$ : 3341, 2937, 1640, 1258.

4-(4-nitrophenyl)-3,6,8-trimethyl-8,9-dihydro-1H-pyrazolo[4',3':5,6]pyrido[2,3-d]pyrimidine-5,7(4H,6H)-dione (Entry 6, Table 2). m.p.: $172-173^{\circ} \mathrm{C}$; IR (KBr, $\left.v_{\max }, \mathrm{cm}^{-1}\right)$ : 3320, 2949, 1671, 1269.

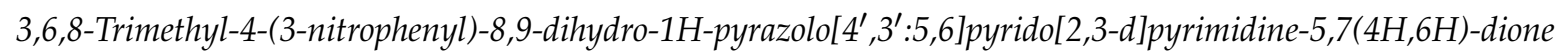
(Entry 7, Table 2). m.p.: $221-223^{\circ} \mathrm{C}$; IR (KBr, $\left.v_{\max }, \mathrm{cm}^{-1}\right): 3325,2951,1643,1271$.

4-(4-Hydroxyphenyl)-3,6,8-trimethyl-8,9-dihydro-1H-pyrazolo[ $\left.4^{\prime}, 3^{\prime}: 5,6\right]$ pyrido[2,3-d]pyrimidine-5,7(4H,6H)dione (Entry 8, Table 2). m.p.: 220-222 ${ }^{\circ} \mathrm{C}$; IR $\left(\mathrm{KBr}, v_{\max }, \mathrm{cm}^{-1}\right): 3318,2963,1617,1280$.

4-(4-(Dimethylamino)phenyl)-3,6,8-trimethyl-8,9-dihydro-1H-pyrazolo[ $\left.4^{\prime}, 3^{\prime}: 5,6\right] p y r i d o[2,3-d] p y r i m i d i n e-5,7$ (4H,6H)-dione (Entry 9, Table 2). m.p.: 222-224 ${ }^{\circ} \mathrm{C}$; IR (KBr, $\left.v_{\max }, \mathrm{cm}^{-1}\right)$ : 3331, 2949, 1631, 1271.

3,6,8-Trimethyl-1,4-diphenyl-8,9-dihydro-1H-pyrazolo[4',3':5,6]pyrido[2,3-d]pyrimidine-5,7(4H,6H)-dione (Entry 10, Table 2). m.p.: $208-210^{\circ} \mathrm{C}$; IR $\left(\mathrm{KBr}, v_{\max }, \mathrm{cm}^{-1}\right): 3315,2936,1650,1241 .{ }^{1} \mathrm{H}-\mathrm{NMR}(400 \mathrm{MHz}$, DMSO-d $\left.d_{6}\right): \delta 1.55\left(\mathrm{~s}, 3 \mathrm{H}, \mathrm{CH}_{3}\right), 2.70\left(\mathrm{~s}, 3 \mathrm{H}, \mathrm{CH}_{3}\right), 2.82\left(\mathrm{~s}, 3 \mathrm{H}, \mathrm{CH}_{3}\right), 5.09(\mathrm{~s}, 1 \mathrm{H}, \mathrm{CH}), 7.42-7.46$ $(\mathrm{t}, J=8.0 \mathrm{~Hz}, 6 \mathrm{H}, \mathrm{Ar}-\mathrm{H}), 7.87-7.89(\mathrm{~d}, J=8.0 \mathrm{~Hz}, 4 \mathrm{H}, \mathrm{Ar}-\mathrm{H}), 11.77(\mathrm{~s}, 1 \mathrm{H}, \mathrm{NH})$.

3,6,8-Trimethyl-4-(4-fluorophenyl)-1-phenyl-8,9-dihydro-1H-pyrazolo[4',3':5,6]pyrido[2,3-d]pyrimidine-5,7 (4H,6H)-dione (Entry 11, Table 2). m.p.: 207-208 ${ }^{\circ} \mathrm{C}$; IR (KBr, $\left.v_{\max }, \mathrm{cm}^{-1}\right)$ : 3324, 2949, 1647, 1270.

4-(4-Chlorophenyl)-3,6,8-trimethyl-8,9-dihydro-1H-pyrazolo[ $\left.4^{\prime}, 3^{\prime}: 5,6\right]$ pyrido[2,3-d]pyrimidine-5,7(4H,6H)-dione (Entry 12, Table 2). m.p.: $200-202{ }^{\circ} \mathrm{C}$; IR $\left(\mathrm{KBr}, v_{\max }, \mathrm{cm}^{-1}\right): 3321,2946,1641,1273 .{ }^{1} \mathrm{H}-\mathrm{NMR}(400 \mathrm{MHz}$, DMSO-d $\left.d_{6}\right): \delta 1.96\left(\mathrm{~s}, 3 \mathrm{H}, \mathrm{CH}_{3}\right), 2.88\left(\mathrm{~s}, 3 \mathrm{H}, \mathrm{CH}_{3}\right), 3.46\left(\mathrm{~s}, 3 \mathrm{H}, \mathrm{CH}_{3}\right), 4.86(\mathrm{~s}, 1 \mathrm{H}, \mathrm{CH}), 7.14-7.19(\mathrm{~m}, 5 \mathrm{H}$, Ar-H), 7.54-7.56 (d, 2H, J = 7.6 Hz, Ar-H), 7.96-7.98 (d, 2H, J = 7.6 Hz, Ar-H), 11.97 (s, 1H, NH); ${ }^{13} \mathrm{C}-\mathrm{NMR}\left(100 \mathrm{MHz}\right.$, DMSO- $\left.d_{6}\right) \delta: 10.91,26.55,29.13,32.69,87.17,101.45,117.40,122.81,124.54,126.33$, $127.91,128.17,129.21,134.52,135.71,145.43,147.38,149.09,161.98$; calcd for $\mathrm{C}_{23} \mathrm{H}_{20} \mathrm{ClN}_{5} \mathrm{O}_{2}$ : $\mathrm{C}, 66.18$; $\mathrm{H}, 4.83 ; \mathrm{N}, 16.78$; found: $\mathrm{C}, 66.34 ; \mathrm{H}, 4.76 ; \mathrm{N}, 16.18$.

4-(4-Bromophenyl)-3,6,8-trimethyl-1-phenyl-8,9-dihydro-1H-pyrazolo[4',3':5,6]pyrido[2,3-d]pyrimidine-5,7 (4H,6H)-dione (Entry 13, Table 2). m.p.: $160-161^{\circ} \mathrm{C}$; IR (KBr, $\left.v_{\max }, \mathrm{cm}^{-1}\right)$ : 3317, 2944, 1641, 1279.

3,6,8-Trimethyl-4-(4-nitrophenyl)-1-phenyl-8,9-dihydro-1H-pyrazolo[4',3':5,6]pyrido[2,3-d]pyrimidine-5,7 (4H,6H)-dione (Entry 14, Table 2). m.p.: 155-157 ${ }^{\circ} \mathrm{C}$; IR (KBr, $\left.v_{\max }, \mathrm{cm}^{-1}\right)$ : 3330, 2954, 1648, 1266.

3,6,8-Trimethyl-1-phenyl-4-(p-tolyl)-8,9-dihydro-1H-pyrazolo[4',3':5,6]pyrido[2,3-d]pyrimidine-5,7(4H,6H)dione (Entry 15, Table 2). m.p.: 205-206 ${ }^{\circ} \mathrm{C}$; IR $\left(\mathrm{KBr}, v_{\max }, \mathrm{cm}^{-1}\right): 3315,2938,1640,1259$.

\section{Conclusions}

In summary, herein, we report a high yielding one-pot synthesis of pyrazolo-[ $\left[4^{\prime}, 3^{\prime}: 5,6\right]$ pyrido[2,3- $\left.d\right]$ pyrimidine-dione derivatives from the condensation of ethyl acetoacetate, hydrazine hydrate or phenyl hydrazine, 1,3-dimethyl barbituric acid, aryl aldehydes and ammonium acetate catalyzed by nano $\mathrm{ZnO}$ and L-proline under triply green conditions, including using green catalysis in water via MCR reaction. The conditions are mild and a wide range of functional groups can be tolerated. Using nano- $\mathrm{ZnO}$ as catalyst offers advantages including simplicity of operation, easy work-up and high yields of products. This work will not only lead to establishing a practical synthetic method but will also expand the versatility of clean organic reactions in water.

Supplementary Materials: Supplementary materials can be accessed at: http://www.mdpi.com/1420-3049/ 21/4/441/s1.

Acknowledgments: Majid M. Heravi gratefully recognizes partial financial support from Iran National Science Foundation (INSF) and Alzahra University.

Author Contributions: M.M.H. and M.D. conceived and designed the experiments, M.D. performed the experiments, $\mathrm{MMH}$ and MD analyzed the data, $\mathrm{MMH}$ contributed reagents/materials/analysis tools, M.M.H. wrote the paper. 
Conflicts of Interest: The authors declare no conflict of interest.

\section{References}

1. Chan, M. World Malaria Report 2011 Summary (Report); World Health Organization: Geneva, Switzerland, 2011.

2. Russell, D.G.; Barry, C.E.; Flynn, J.L. Tuberculosis: What We Don't Know Can, and Does, Hurt Us. Science 2010, 328, 852-856. [CrossRef] [PubMed]

3. Ballell, L.; Field, R.A.; Duncan, K.; Young, R.J. New Small-Molecule Synthetic Antimycobacterials. Antimicrob. Agents Chemother. 2005, 49, 2153-2163. [CrossRef] [PubMed]

4. Cegielski, J.P.; Chin, D.P.; Espinal, M.A.; Frieden, T.R.; Cruz, R.R.; Talbot, E.A.; Weil, D.E.C.; Zaleskis, R.; Raviglione, M.C. The global tuberculosis situation. Progress and problems in the 20th century, prospects for the 21st century. Infect. Dis. Clin. N. Am. 2002, 16, 1-58. [CrossRef]

5. Selvam, T.P.; James, C.R.; Dniandev, P.V.; Valzita, S.K. A mini review of pyrimidine and fused pyrimidine marketed Drugs. Res. Pharm. 2012, 2, 1-9.

6. Agarwal, A.; Srivastava, K.; Puri, S.K.; Chauhan, P.M. Synthesis of 2,4,6-trisubstituted pyrimidines as antimalarial agents. Bioorg. Med. Chem. 2005, 13, 4645-4650. [CrossRef] [PubMed]

7. Matloobi, M.C.; Kappe, O. Microwave-Assisted Solution- and Solid-Phase Synthesis of 2-Amino-4arylpyrimidine Derivatives. J. Comb. Chem. 2007, 9, 275-284. [CrossRef] [PubMed]

8. Gadhachanda, V.R.; Wu, B.; Wang, Z.; Kuhen, K.L.; Caldwell, J.; Zondler, H.; Walter, H.; Havenhand, M.; He, Y. Antibacterial 5'-O-(N-dipeptidyl)-sulfamoyladenosines. Bioorg. Med. Chem. 2007, 17, 260-265. [CrossRef] [PubMed]

9. Chang, L.C.W.; Spanjersberg, R.F.; von Frijtag Drabbe Kunzel, J.K.; Mulder-Krieger, T.; van den Hout, G.; Beukers, M.W.; Brussee, J.; Jzerman, A.P. 2,4,6-Trisubstituted Pyrimidines as a New Class of Selective Adenosine A1 Receptor Antagonists. J. Med. Chem. 2004, 47, 6529-6540. [CrossRef] [PubMed]

10. Capdeville, R.; Buchdunger, E.; Zimmermann, J.; Matter, A. Glivec (STI571, imatinib), a rationally developed, targeted anticancer. Nat. Rev. Drug Discov. 2002, 1, 493-502. [CrossRef] [PubMed]

11. Zaki, M.E.A.; Soliman, H.A.; Hiekal, O.A.; Rashad, A.E.Z. Pyrazolopyranopyrimidines as a Class of Anti-Inflammatory Agents. Z. Naturforschung 2006, 61c, 1-5. [CrossRef]

12. Ozeki, K.; Ichikawa, T.; Takehara, H.; Tanimura, K.; Sato, M.; Yaginuma, H. Studies on antiallergy agents. III. Synthesis of 2-anilino-1,6-dihydro-6-oxo-5-pyrimidine carboxylic acids and related compounds. Chem. Pharm. Bull. 1989, 37, 1780-1787. [CrossRef] [PubMed]

13. Hulme, C.; Gore, V. "Multi-component Reactions: Emerging Chemistry in Drug Discovery" "from Xylocain to Crixivan". Curr. Med. Chem. 2003, 10, 51-80. [CrossRef] [PubMed]

14. Dömling, A.; Ugi, I. Multicomponent Reactions with Isocyanides. Angew. Chem. Int. Ed. 2000, 39, 3169-3210. [CrossRef]

15. Shaabani, A.; Seyyedhamzeh, M.; Maleki, A.; Behnam, M.; Rezazadeh, F. Synthesis of fully substituted pyrazolo[3,4-b]pyridine-5-carboxamide derivatives via a one-pot four-component reaction. Tetrahedron Lett. 2009, 50, 2911-3020. [CrossRef]

16. Sunderhaus, J.D.; Martin, S.F. Applications of Multicomponent Reactions to the Synthesis of Diverse Heterocyclic Scaffolds. Chem. Eur. J. 2009, 15, 1300-1308. [CrossRef] [PubMed]

17. Li, C.-J. Organic reactions in aqueous media-With a focus on carbon-carbon bond formation. Chem. Rev. 1993, 93, 2023-2035. [CrossRef]

18. Lindstrom, U.M. Stereoselective Organic Reactions in Water. Chem. Rev. 2002, 102, 2751-2772. [CrossRef] [PubMed]

19. Breslow, R. Hydrophobic effects on simple organic reactions in water. Acc. Chem. Res. 1991, 24, $159-164$. [CrossRef]

20. Li, C.J. Organic Reactions in Aqueous Media with a Focus on Carbon-Carbon Bond Formations: A Decade Update. Chem. Rev. 2005, 105, 3095-3165. [CrossRef] [PubMed]

21. Kobayashi, S. Asymmetric catalysis in aqueous media. Pure Appl. Chem. 2007, 79, 235-245. [CrossRef]

22. Narayan, S.; Muldoon, J.; Glynn, M.G.; Fokin, V.V.; Kolb, H.C.; Sharpless, K.B. “On water”: Unique reactivity of organic compounds in aqueous suspension. Angew. Chem. Int. Ed. 2005, 44, 3275-3279. [CrossRef] [PubMed] 
23. Rothenberg, G. Catalysis: Concepts and Green Applications; Wiley-VCH: Weinheim, Germany, 2008.

24. Rostamnia, S.; Doustkhah, E. Nanoporous silica-supported organocatalyst: A heterogeneous and green hybrid catalyst for organic transformations. RSC Adv. 2014, 4, 28238-28248. [CrossRef]

25. Bazgira, A.; Khanaposhtani, M.M.; Soorki, A.A. One-pot synthesis and antibacterial activities of pyrazolo[40,30:5,6]pyrido[2,3-d]pyrimidine-dione derivatives. Bioorg. Med. Chem. Lett. 2008, 18, 5800-5803. [CrossRef] [PubMed]

26. Satasia, S.P.; Kalaria, P.N.; Raval, D.K. Catalytic regioselective synthesis of pyrazole based pyrido[2,3-d]pyrimidine-diones and their biological evaluation. Org. Biomol. Chem. 2014, 12, 1751-1758. [CrossRef] [PubMed]

27. Heravi, M.M.; Alishiri, T. Chapter One-Dimethyl Acetylenedicarboxylate as a Building Block in Heterocyclic Synthesis. Adv. Heterocycl. Chem. 2014, 113, 1-66.

28. Heravi, M.M.; Talaei, B. Chapter Four-Ketenes as Privileged Synthons in the Syntheses of Heterocyclic Compounds. Part 1: Three- and Four-Membered Heterocycles. Adv. Heterocycl. Chem. 2014, 113, 143-244.

29. Heravi, M.M.; Khaghaninejad, S.; Nazari, N. Chapter Five-Bischler-Napieralski Reaction in the Syntheses of Isoquinolines. Adv. Heterocycl. Chem. 2014, 112, 183-234.

30. Heravi, M.M.; Zadsirjan, V. Chapter Five-Recent Advances in the Synthesis of Benzo[b]furans. Adv. Heterocycl. Chem. 2015, 117, 261-376.

31. Heravi, M.M.; Talaei, B. Chapter Three-Ketenes as Privileged Synthons in the Syntheses of Heterocyclic Compounds Part 2: Five-Membered Heterocycles. Adv. Heterocycl. Chem. 2015, 114, 147-225.

32. Heravi, M.M.; Fathi Vavsari, V. Chapter Two-Recent Advances in Application of Amino Acids: Key Building Blocks in Design and Syntheses of Heterocyclic Compounds. Adv. Heterocycl. Chem. 2015, 114, 77-145.

33. Khaghaninejad, S.; Heravi, M.M. Chapter Three-Paal-Knorr Reaction in the Synthesis of Heterocyclic Compounds. Adv. Heterocycl. Chem. 2014, 111, 95-146.

34. Heravi, M.M.; Khaghaninejad, S.; Mostofi, M. Chapter One-Pechmann Reaction in the Synthesis of Coumarin Derivatives. Adv. Heterocycl. Chem. 2014, 112, 1-50.

35. Mirsafaei, R.; Heravi, M.M.; Ahmadi, S.; Moslemin, M.H.; Hosseinnejad, T. In situ prepared copper nanoparticles on modified KIT-5 as an efficient recyclable catalyst and its applications in click reactions in water. J. Mol. Catal. A Chem. 2015, 402, 100-108. [CrossRef]

36. Nemati, F.; Heravi, M.M.; Elhampour, A. Magnetic nano- $-\mathrm{Fe}_{3} \mathrm{O}_{4} @ \mathrm{TiO}_{2} / \mathrm{Cu}_{2} \mathrm{O}$ core-shell composite: An efficient novel catalyst for the regioselective synthesis of 1,2,3-triazoles using a click reaction. RSC Adv. 2015, 5, 45775-45784. [CrossRef]

37. Nemati, F.; Heravi, M.M.; Saeedi, R. Nano-Fe ${ }_{3} \mathrm{O}_{4}$ Encapsulated-Silica Particles Bearing Sulfonic Acid Groups as a Magnetically Separable Catalyst for Highly Efficient Knoevenagel Condensation and Michael Addition Reactions of Aromatic Aldehydes with 1,3-Cyclic Diketones. Chin. J. Catal. 2012, 33, 1825-1831. [CrossRef]

38. Heravi, M.M.; Alishiri, T. Application of Nanomaterials in Heterocyclic Chemistry. Heterocycles 2012, 85, 545-586. [CrossRef]

39. Hashemi, E.; Beheshtiha, S.Y.; Ahmadi, S.; Heravi, M.M. In situ prepared CuI nanoparticles on modified poly(styrene-co-maleic anhydride): An efficient and recyclable catalyst for the azide-alkyne click reaction in water. Transit. Met. Chem. 2014, 39, 593-601. [CrossRef]

40. Heravi, M.M.; Hashemi, E.; Beheshtiha, S.Y.; Kamjou, K.; Toolabi, M. PdCl 2 on modified poly(styrene-co-maleic anhydride): A highly active and recyclable catalyst for the Suzuki-Miyaura and Sonogashira reactions. J. Mol. Catal. A Chem. 2014, 394, 74-82. [CrossRef]

41. Heravi, M.M.; Daraie, M.; Behbahani, F.K.; Malakooti, R. Green and Novel Protocol for One-Pot Synthesis of $\beta$-Acetamido Carbonyl Compounds Using $\mathrm{Mn}(\mathrm{bpdo})_{2} \mathrm{Cl}_{2} / \mathrm{MCM}-41$ Catalyst. Synth. Commun. 2010, 40, 1180-1186. [CrossRef]

42. Daraie, M.; Beheshtiha, Y.S.; Heravi, M.M. Synthesis of spirochromene derivatives catalyzed by Mn(bpyo) 2 /MCM-41 in water. Monatshefte Chem. 2015, 146, 191-198. [CrossRef]

43. Heravi, M.M.; Daraie, M. Heterogeneous catalytic three-component one-pot synthesis of novel $8 H$-[1,3]dioxolo[4,5- $g$ ] chromenes by basic alumina in water. Mon. Chem. 2014, 145, 1479-1482. [CrossRef]

44. Heravi, M.M.; Daraie, M. $\mathrm{Mn}(\mathrm{pbdo})_{2} \mathrm{Cl}_{2} / \mathrm{MCM}-41$ as a green catalyst in multi-component syntheses of some heterocycles. Res. Chem. Intermed. 2015, 41, 1-10. [CrossRef] 
45. Heravi, M.M.; Khorasani, M.; Derikvand, F.; Bamoharram, F.F. Highly efficient synthesis of coumarin derivatives in the presence of $\mathrm{H}_{14}\left[\mathrm{NaP}_{5} \mathrm{~W}_{30} \mathrm{O}_{110}\right]$ as a green and reusable catalyst. Catal. Commun. 2007, 8, 1886-1890. [CrossRef]

46. Heravi, M.M.; Bakhtiari, K.; Taheri, S. $\mathrm{KHSO}_{4}$ : A catalyst for the chemo-selective preparation of 1,1-diacetates from aldehydes under solvent-free conditions. Green Chem. 2005, 7, 867-869. [CrossRef]

47. Saeedi, M.; Heravi, M.M.; Beheshtiha, S.Y. One-pot three-component synthesis of the spiroacenaphthylene derivatives. Tetrahedron 2010, 66, 5345-5348. [CrossRef]

48. Heravi, M.M.; Baghernejad, B.; Oskooie, H.A. A novel three-component reaction for the synthesis of $\mathrm{N}$-cyclohexyl-3-aryl-quinoxaline-2-amines. Tetrahedron Lett. 2009, 50, 767-769. [CrossRef]

49. Heravi, M.M.; Baghernejad, B.; Oskooie, H.A. A novel and facile synthesis of 2-(cyclohexylamino)6,7-dihydro-3-aryl-1H-indole-4(5H)-ones via a one-pot multi-component reaction. Tetrahedron Lett. 2009, 49, 6106-6103. [CrossRef]

50. Heravi, M.M.; Moghimi, S. An efficient synthesis of thiazol-2-imine derivatives via a one-pot, three-component reaction. Tetrahedron Lett. 2012, 53, 392-394. [CrossRef]

51. Heravi, M.M.; Mousavizadeh, F.; Ghobadi, N.; Tajbakhsh, M. A green and convenient protocol for the synthesis of novel pyrazolopyranopyrimidines via a one-pot, four-component reaction in water. Tetrahedron Lett. 2014, 55, 1226-1228. [CrossRef]

52. Heravi, M.M.; Behbahani, F.K.; Daraie, M.; Oskooie, H.A. $\mathrm{Fe}\left(\mathrm{ClO}_{4}\right)_{3} \cdot 6 \mathrm{H}_{2} \mathrm{O}$ : A mild and efficient catalyst for one-pot three component synthesis of $\beta$-acetamido carbonyl compounds under solvent-free conditions. Mol. Divers. 2009, 13, 375-378. [CrossRef] [PubMed]

53. Heravi, M.M.; Tajbakhsh, M.; Ahmadi, A.N.; Mohajerani, B. Zeolites. Efficient and Eco-friendly Catalysts for the Synthesis of Benzimidazoles. Mon. Chem. 2006, 137, 175-179. [CrossRef]

54. Heravi, M.M.; Sadjadi, S.; Oskooie, H.A.; Hekmat Shoar, R.; Bamoharram, F.F. Heteropolyacids as Green and Reusable Catalysts for the Synthesis of 3,1,5-Benzoxadiazepines. Molecules 2007, 12, 255-262. [CrossRef] [PubMed]

55. Shaabani, A.; Ghadari, R.; Ghasemi, S.; Pedarpour, M.; Rezayan, A.H.; Sarvary, A.; Seik Weng, N.G. Novel One-Pot Three- and Pseudo-Five-Component Reactions: Synthesis of Functionalized Benzo[g]- and Dihydropyrano[2,3-g]chromene Derivatives. J. Comb. Chem. 2009, 11, 956-959. [CrossRef] [PubMed]

56. Lu, Z.; Xiao, J.; Wang, D.; Li, Y. An Efficient One-Pot Five-Component Tandem Sequential Approach for the Synthesis of Pyranopyrazole Derivatives via Suzuki Coupling and Multicomponent Reaction. Asian J. Org. Chem. 2015, 4, 487-492. [CrossRef]

57. Fu, L.; Feng, X.; Zhang, J.; Hu, J.-D.; Xun, Z.; Wang, J.-J.; Huang, Z.-B.; Shi, D.-Q. Highly efficient construction of a bridged pentacyclic skeleton via a six-component domino reaction under microwave irradiation. Green Chem. 2015, 17, 1535-1545. [CrossRef]

58. Basyouni, W.M.; El-Bayouki, K.A.M.; Tohamy, W.M.; Abbas, S.Y. Silica Sulfuric Acid: An Efficient, Reusable, Heterogeneous Catalyst for the One-Pot, Five-Component Synthesis of Highly Functionalized Piperidine Derivatives. Synth. Commun. 2015, 45, 1073-1081. [CrossRef]

59. Dabiri, M.; Salehi, P.; Fakharian, M.; Movahed, S.K.; MaGee, D. New Convenient Five-Component One-Pot Synthesis of 3-Alkyl-6-amino-1,4-dihydro-4-\{[(1,2,3-triazol-4-yl)methoxy]phenyl\}pyrano[2,3-c]pyrazole5-carbonitrile Derivatives. Helv. Chim. Acta 2015, 98, 633-641. [CrossRef]

60. Nikbakht, A.; Ramezanpour, S.; Balalaie, S.; Rominger, F. Efficient and stereoselective synthesis of $\alpha$-hydrazino tetrazoles through a pseudo five-component domino reaction. Tetrahedron 2015, 71, 6790-6795. [CrossRef]

61. Teimouri, M.B.; Moghaddam, P.A.; Golbaghi, G. Pseudo-Five-Component Reaction between 3-Formylchromones, Meldrum's Acid, Isocyanides and Primary Arylamines: Diversity-Oriented Synthesis of Novel Chromone-Containing Peptidomimetics. ACS Comb. Sci. 2011, 13, 659-666. [CrossRef] [PubMed]

62. Kimata, A.; Nakagawa, H.; Ohyama, R.; Fukuuchi, T.; Ohta, S.; Suzuki, T.; Miyata, N. New Series of Antiprion Compounds: Pyrazolone Derivatives Have the Potent Activity of Inhibiting Protease-Resistant Prion Protein Accumulation. J. Med. Chem. 2007, 50, 5053-5056. [CrossRef] [PubMed]

63. Antre, R.V.; Cendilkumar, A.; Goli, D.; Andhale, G.S.; Oswal, R. Microwave assisted synthesis of novel pyrazolone derivatives attached to a pyrimidine moiety and evaluation of their anti-inflammatory, analgesic and antipyretic activities. J. Saudi Pharm. J. 2011, 19, 233-243. [CrossRef] [PubMed] 
64. Mecadon, H.; Rohman, M.R.; Kharbangar, I.; Laloo, B.M.; Kharkongor, I.; Rajbangshi, M.; Myrboh, B. L-Proline as an efficicent catalyst for the multi-component synthesis of 6-amino-4-alkyl/aryl-3-methyl-2,4dihydropyrano[2,3-c]pyrazole-5-carbonitriles in water. Tetrahedron Lett. 2011, 52, 3228-3231. [CrossRef]

65. Mukhopadhyay, C.; Tapaswi, P.K.; Butcher, R.J. 1-Proline-catalyzed one-pot expeditious synthesis of highly substituted pyridines at room temperature. Tetrahedron Lett. 2010, 51, 1797-1802. [CrossRef]

66. Hernández, J.G.; García-López, V.; Juaristi, E. Solvent-free asymmetric aldol reaction organocatalyzed by (S)-proline-containing thiodipeptides under ball-milling conditions. Tetrahedron 2012, 68, 92-97. [CrossRef]

67. List, B. Proline-catalyzed asymmetric reactions. Tetrahedron 2002, 58, 5573-5590. [CrossRef]

Sample Availability: Not available.

(C) 2016 by the authors; licensee MDPI, Basel, Switzerland. This article is an open access article distributed under the terms and conditions of the Creative Commons by Attribution (CC-BY) license (http://creativecommons.org/licenses/by/4.0/). 